The Bulletin of Symbolic Logic

Volume 5, Number 3, Sept. 1999

\title{
IN MEMORIAM: ALBERT G. DRAGALIN
}

1941-1998

Albert G. Dragalin, an important figure in the development of mathematical logic in the former Soviet Union, passed away from a sudden heart attack in Debrecen (Hungary) on December 18, 1998, at the age of 57 . He was born on April 10, 1941 in Morzchevec (in the Arkhangelsk region in northern Russia). A. Dragalin received his Master's Degree in 1963 and his Ph. D. in 1968 under A. A. Markov Jr. from the Department of Mathematics and Mechanics of Moscow State University. After joining the Chair of Mathematical Logic in the same Department in 1968, he established several graduate courses in mathematical logic, especially in proof theory, intuitionistic logic, and axiomatic set theory. Almost every year he introduced a new course. His seminar on proof theory was for many years one of the most important centers of activity in mathematical logic in Moscow. It attracted many young mathematicians. He was a very lively and enthusiastic teacher with broad views on logic and mathematics. Practically every undergraduate or graduate student interested in mathematical logic was influenced by him, even if he was not always a formal advisor. Dragalin supervised a large number of Master students and more than a dozen $\mathrm{Ph}$. $\mathrm{D}$. disserations in mathematical logic.

He played an important part in establishing a course in mathematical logic for all mathematics majors at Moscow State University. This course was first taught by A. N. Kolmogorov. Dragalin coauthored with A. N. Kolmogorov two textbooks [7, 8] for this course.

Dragalin's independent personality did not get along well with the Soviet realities in general and the depressing situation in Soviet mathematical logic in particular.

In 1983 in the middle of his academic career he left Moscow for Debrecen, Hungary, with his second wife Svetlana Buzàsi, who was a Hungarian mathematician of Russian origin. In Hungary, Albert struggled to establish himself in a new cultural and scientific environment and eventually succeeded. He joined the Computer Center of the Lajos Kossuth University at Debrecen and moved to the Department of Computer Science of the same university in 1990. Dragalin received his Doctor of Sciences Degree in mathematics from the Hungarian Academy of Sciences in 1988. In 1993, he became chair of the Department of Computer Science of the Lajos Kossuth University. 
In 1991 his second wife died and four years later Albert married Elena Chernaya, an active logician from Moscow. They had a daughter born in 1996.

The research interests of Dragalin were broad. He was a prominent member of the Russian (Markov) constructivist school and many of his papers contained constructivization of important results whose proofs, and sometimes even statements, transcended established boundaries. These papers contained essentially new results and always provided starting points for his students' work. For example [1] sketched a Tait-style computability proof for simply typed lambda terms with primitive recursion: Tait's own proof avoided difficulties with variables by using combinators instead of lambdaabstraction. In a series of papers, Dragalin adapted Schütte-style infinitary cut-elimination and its proof-theoretic uses, including the admissibility of Markov's rule. A. Dragalin also promoted the use of advanced techniques in set theory and edited translations of several monographs into Russian. Another important construction (obtained in [3] independently of H. Friedman) is an elementary translation "inverse" to the famous negative translation for proofs of purely existential formulas. It provides an elementary admissibility proof of Markov's rule $\neg \neg \exists x A / \exists x A, A$ quantifier-free, for intuitionistic systems and hence shows that the provably recursive functions coincide for a wide class of classical and intuitionistic systems.

A series of papers was devoted to the notion of choice sequence and related realizability notions (cf. [2]).

A. Dragalin had a deep interest in normalization results for derivations in logical systems. One of his constructions frequently used in the literature is the strong normalization proof [4] for Gentzen-style (antecedent-succedent) first order systems: every sequence of cut-elimination transformations terminates, if they do not permute adjacent cuts. A less well-known result of 1977 (cf. [6]) concerns the system Dragalin called the theory of definable sets of natural numbers. It is a second order arithmetic with the $\omega$-rule for natural numbers and the second order rules:

$$
\frac{\phi[X / t], \Gamma \Longrightarrow \Delta}{\forall X \phi, \Gamma \Longrightarrow \Delta} \quad \frac{\ldots \Gamma \Longrightarrow \Delta, \phi[x / t] \ldots \text { for all } t}{\Gamma \Longrightarrow \Delta, \forall x \phi}
$$

where $t$ ranges over closed abstracts $\lambda y \psi$, i.e., $\psi$ does not have any free variables except $y$. A. Dragalin proved a cut-elimination theorem for this system using an imaginative combination of Tait-Takahashi completion of semivaluations and a constructibility technique. The latter is used to retrieve the definitions of the sets employed in the construction.

Another cycle of papers was devoted to elegant intuitionistic versions of model-theoretic proofs of completeness theorems for strong classical and intuitionistic theories. The work on automated deduction [5] exemplifies more applied lines of research. 
Many of these investigations were presented in a short book [4], which is a frequently cited reference in the areas Dragalin touched.

An important aspect of his activity was a collaboration with researchers in philosophical logic which helped both sides defend their right to do reasonable work against dogmatic attacks.

Colleagues and friends remember Dragalin as a person of great integrity and dignity. One could always rely on his word. He loved life and behind his rather stern appearance one could sense a warm and kind soul.

Dragalin was full of plans for the future and did not anticipate that death was approaching. He did not sleep his last night. He worked late at his desk and then talked with his wife about God, life and the future. His death was sudden and occurred at 5 A.M. He died with an expression of amazement on his face.

Dragalin is survived by his ex-wife from his first marriage, his widow and two children.

\section{S. Artemov, B. Kushner, G. Mints, E. Nogina, and A. Troelstra}

\section{REFERENCES}

[1] A. G. Dragalin, The computability of primitive recursive terms of finite type, and primitive recursive realization, Seminars in mathematics, vol. 8, Plenum Publishers, 1970, Russian original 1968.

[2] - Constructive models of intuitionistic choice sequences (Russian), Studies in formalized languages and nonclassical logics, Izdat. "Nauka”, Moscow, 1974, pp. 214-252.

[3] - New kinds of realizability and the Markov rule, Soviet Mathematics, Doklady, vol. 251 (1980), no. 3, pp. 534-537.

[4] — Mathematical intuitionism, introduction to proof theory, Translation of Mathematical Monographs, no. 67, American Mathematical Society, Providence, RI, 1988, Russian original 1979.

[5] - Some trends in automated reasoning, Journal of Information Processing Cybernet., vol. 25 (1989), no. 11-12, pp. 607-618.

[6] - Cut-elimination in the theory of definable sets of natural numbers, Publicationes Mathematicae, Debrecen, vol. 51 (1997), no. 1/2, pp. 153-164, Russian version 1977.

[7] A. N. Kolmogorov and A. G. Dragalin, Introduction into mathematical logic (Russian), Moscow University, Moscow, 1982.

[8] — Mathematical logic, advanced chapters (Russian), Moscow University, Moscow, 1984. 